\title{
Microstructures and Thermoelectric Properties of Sintered Misfit-Layered Cobalt Oxide.
}

Takao Morimura ${ }^{1,2}$, Takahiro Yamaguchi ${ }^{1}$, Takuya Kojima ${ }^{1}$, Noriki Matsuya ${ }^{1}$, Shin-ichiro Kondo ${ }^{1}$, Hiromichi Nakashima ${ }^{1}$

1. - Graduate School of Engineering, Nagasaki University, 1-14 Bunkyo-machi, Nagasaki 852-8521, Japan. 2.—e-mail: tmori@nagasaki-u.ac.jp

\begin{abstract}
Misfit-layered cobalt oxide $\mathrm{Ca}_{3} \mathrm{Co}_{4} \mathrm{O}_{9}$ is considered to be a prospective material for thermoelectric conversion. The thermoelectric properties are anisotropic owing to its anisotropic crystal structure. The crystal has preferred thermoelectric properties along $a-b$ plane. Therefore, the thermoelectric properties are improved and controlled by degree of orientation of the sintered sample. In the present work, Sr doped misfit cobalt oxides $\mathrm{Ca}_{2.7} \mathrm{Sr}_{0.3} \mathrm{Co}_{4} \mathrm{O}_{9}$ was prepared by solid-phase reaction, sequent uniaxial compression molding and sintering at $1173 \mathrm{~K}$. Seebeck coefficient $\alpha$, electrical resistivity $\rho$, and dimensionless figure of merit $Z T$ were measured with change of the compression pressure in the uniaxial molding. $\alpha, \rho$, and $Z T$ as functions of the degree of orientation and the relative density are experimentally clarified and explained by the calculations of the complex model.
\end{abstract}

Key words: Seebeck coefficient; dimensionless figure of merit; degree of orientation; Lotgering factor; Harman method 


\section{Introduction}

The misfit cobalt oxides $\mathrm{Ca}_{3} \mathrm{Co}_{4} \mathrm{O}_{9}$ are good representatives of thermoelectric materials because of the high dimensionless figure of merit $Z T$ and superior thermal stability at high temperatures. The unit cell is monoclinic and consists of alternate stacks of a triple rocksalt $\mathrm{Ca}_{2} \mathrm{CoO}_{3}$ layer (RS) and a $\mathrm{CdI}_{2}$-type $\mathrm{CoO}_{2}$ layer along the $c$-axis. The two types of layers have the same $a, c$ and $\beta$ lattice parameters but different $b_{\mathrm{CoO} 2}$ and $b_{\mathrm{RS}}$ parameters. ${ }^{1,2}$ The $\mathrm{CoO}_{2}$ layer are considered responsible for electrical transport, while rocksalt layers are for charge reserve that supplies charge carriers into $\mathrm{CoO}_{2}$ layers. The thermoelectric properties are anisotropic owing to this anisotropic crystal structure and it has preferred thermoelectric properties along $a-b$ plane. ${ }^{3-6}$ Therefore, the thermoelectric properties of the sintered sample are improved by the incease in degree of orientation, which is dependent on the compression pressure in the uniaxial molding. On the other hand, the addition of $\mathrm{Sr}$ to $\mathrm{Ca}_{3} \mathrm{Co}_{4} \mathrm{O}_{9}$ can effectively improve the thermoelectric properties owing to the large solution limit of Sr in the RS layer. ${ }^{7-13}$ In the present work, Sr doped misfit cobalt oxides $\mathrm{Ca}_{2.7} \mathrm{Sr}_{0.3} \mathrm{Co}_{4} \mathrm{O}_{9}$ was prepared by solid-phase reaction, sequent uniaxial compression molding and sintering at $1173 \mathrm{~K}$. The effect of the compression pressure in the uniaxial molding on the microstructures and thermoelectric properties was investigated by X-ray diffraction (XRD) and by measurments of the Seebeck coefficient $\alpha$, electrical conductivity $\rho$, and ZT. The dependences of $\alpha$ and $\rho$ on the degree of orientation $f$ and relative density $d$ were experimentally revealed. Furthermore, the dependences were calculated and explained by the complex model, in which the sintered sample is composed of many $\mathrm{Ca}_{3} \mathrm{Co}_{4} \mathrm{O}_{9}$ elements with different orientations and void elements.

\section{Experimental procedure}

$\mathrm{CaCO}_{3}, \mathrm{Co}_{3} \mathrm{O}_{4}$, and $\mathrm{SrCO}_{3}$ powders were weighed according to the proportions of the $\mathrm{Ca}_{2 .} \mathrm{Sr}_{0.3} \mathrm{Co}_{4} \mathrm{O}_{9}$ composition. The powders were thoroughly mixed with acetone in an agate mortar, heated at $1123 \mathrm{~K}$ for $20 \mathrm{~h}$ in an alumina crucible in atmosphere, and then slowly cooled to room temperature. The products were ground into powder $(\sim 100 \mu \mathrm{m})$, molded at uniaxial compression of 150-900 MPa, and then sintered at 
$1173 \mathrm{~K}$ for $20 \mathrm{~h}$ in atmosphere to fabricate pellet samples. The compression direction was perpendicular to the basal plane of the pellets.

The Seebeck coefficient $\alpha \exp$ was determined by measuring the voltage at both ends of the pellets, which is caused by the difference in temperature. Electrical resistivity $\rho_{\exp }$ was measured using the four-point probe method at room temperature. The dimensionless figure of merit $Z T$ was measured by the Harman method.$^{14}$ The measurements of the thermoelectric properties were performed parallel to the basal plane of the pellet samples in atmosphere. For the XRD samples, the pellets were ground into powder ( $\sim 50 \mu$ ). The XRD patterns were obtained by using a Rigaku Rint-2200VL and Cu $\mathrm{K}_{\alpha}$ radiation for the powder and pellet samples. The degrees of orientation $f$ of the pellet samples were estimated by using the XRD peak intensities as Lotgering factor. ${ }^{15}$ The relative densities $d$ of the pellet samples were obtained by Archimedian method.

\section{Results and discussion}

Figure 1 (a) shows $\alpha_{\exp }(\bigcirc)$ and $\rho_{\exp }(\bigcirc)$ measured at room temperature as a function of the compression pressure in the uniaxial molding. $\alpha_{\exp }$ was positive, indicating p-type conductivity. $\alpha_{\exp }$ slightly increases and $\rho_{\exp }$ decreases with the increase in the compression pressure. These results are similar to the previous report [4] although the preparing process is different. These may be attributed to the increase in $f$ and $d$. $f$ increases with the compression pressure because the $a-b$ planes of $\mathrm{Ca}_{3} \mathrm{Co}_{4} \mathrm{O}_{9}$ are aligned parallel to the basal plane of the pellet as shown in the SEM images and the pole figures of Ref. [4]. $d$ increases with the compression pressure because of extinction of the void in the pellet. Fig. 1 (b) shows $Z T(\bigcirc)$ and the power factor $P F(\bigcirc)$ at room temperature as a function of the compression pressure. They increase with the compression pressure through the increase in $\alpha_{\exp }$ and the decrease in $\rho_{\exp }$.

Figure 2 shows $Z T$ as a function of the measured temperature for various compression pressures. ZT increases with the measured temperature. The trend that $Z T$ increases with the compression pressure holds for high temperature. 
Figures 3 (a) shows the X-ray diffraction patterns of $\mathrm{Ca}_{2.7} \mathrm{Sr}_{0.3} \mathrm{Co}_{4} \mathrm{O}_{9}$ for the powder sample and the pellet samples molded at 150-900 MPa. Most diffraction peaks are indexed as the misfit cobalt oxide $\mathrm{Ca}_{3} \mathrm{Co}_{4} \mathrm{O}_{9}$ phase. The peak intensities other than $00 \mathrm{l}$ peaks decrease with the increase in the compression pressure because $a-b$ planes of the cleaved $\mathrm{Ca}_{3} \mathrm{Co}_{4} \mathrm{O}_{9}$ particles are aligned parallel to the basal plane of the pellet. $f$ is quantitatively estimated as the Lotgering factor: ${ }^{15}$

$f=\frac{P-P_{0}}{1-P_{0}}$

$P=\frac{\sum_{l} I(00 l)}{\sum_{h k l} I(h k l)}$

where $I(00 l)$ and $I(h k l)$ indicate the integrated intensities of $00 l$ and $h k l$ peaks, respectively. $P_{0}$ and $P$ correspond to the powder and the pellet samples, respectively. Fig. 3 (b) shows $f$ and $d$ as a function of the compression pressure. $f$ increases with the increase in the compression pressure. $d$ also increases because of the extinction of the void.

$\alpha_{\text {exp }}$ and $\rho_{\text {exp }}$ measured at room temperature are shown as $\bigcirc$ and $\bigcirc$ (and smoothed solid lines) in Fig. 4 (a) and (b) as a function of $f$ and $d$, respectively. $\alpha \exp$ slightly increases and $\rho_{\exp }$ decreases with the increase in $f$ and $d$ owing to the alignment of the cleaved $\mathrm{Ca}_{3} \mathrm{Co}_{4} \mathrm{O}_{9}$ particles and/or the extinction of the voids.

In order to clarify the effect of $f$ and $d$ on $\alpha_{\exp }$ and $\rho_{\text {exp }}$, the calculations based on complex model ${ }^{16,17}$ were performed. In this model, the sample was assumed to be the complex composed of A and B elements as shown in Fig. 5. When the volume fractions of the $\mathrm{A}$ and $\mathrm{B}$ elements are defined to be $C_{\mathrm{A}}$ and $C_{\mathrm{B}}$ $\left(C_{\mathrm{A}}+C_{\mathrm{B}}=1\right)$, the Seebeck coefficient $\alpha_{\text {comp }}$ and the electrical resistivity $\rho_{\text {comp }}$ along the measured direction $y$ for the complex were formulated by the combination of serial and parallel alignments of the A and B elements as ${ }^{16,17}$

$$
\begin{aligned}
& \rho_{\text {comp }}=\rho_{A}(1-K)+\frac{\rho_{A} \rho_{B}}{\rho_{B}\left(1-\frac{C_{B}}{K}\right)+\rho_{A} c_{B} / K} K \\
& \alpha_{\text {comp }}=\alpha_{A}(1-K)+\frac{\alpha_{A} \rho_{B}\left(1-\frac{C_{B}}{K}\right)+\alpha_{B} \rho_{A} C_{B} / K}{\rho_{B}\left(1-\frac{C_{B}}{K}\right)+\rho_{A} c_{B} / K} K^{16,17}
\end{aligned}
$$


Here, $\alpha_{\mathrm{A}}, \alpha_{\mathrm{B}}, \rho_{\mathrm{A}}$ and $\rho_{\mathrm{B}}$ are the Seebeck coefficients and the electrical resistivities for A and B elements, respectively. $K$ is the ratio of the $x$-z layers including the B element to the all $x$ - $z$ layers perpendicular to $y$ axis. $C_{\mathrm{B}} / K$ is the averaged fraction of $\mathrm{B}$ element within the $x-z$ layer including both of $\mathrm{A}$ and $\mathrm{B}$ elements. In Eqs. (1) and (2), the first terms of the right hand sides indicate the electrical resistivity and the Seebeck coefficient for the $x-z$ layers including only A element, respectively. The second terms of the right hand sides indicate those for the $x-z$ layers including both of A and B elements. The equations indicate that two kinds of layers are serially aligned along the measured direction $y$. If the B element is assumed to be randomly distributed in the cuboid pellet with the width $w$, length $l$, and height $h, K$ is estimated as $C_{B}^{\frac{l}{w+l+h}}$. This is because the boundary conditions are satisfied as $K=1$ for $h, w 》 1$ and $l \ll 1$ and as $K=C_{\mathrm{B}}$ for $h, w$ 《1, and $l 》 1$.

In the present case, we assumed the sintered pellet samples as the complex of three kinds of elements: the void and two kinds of oxide elements whose $a-b$ planes are aligned parallel to and perpendicular to the measured direction $(y)$. At the first step, the sintered pellets are simply assumed to be the complex composed of the oxide and the void elements. The volume fractions of the oxide and the void elements are defined to be $C_{\mathrm{ox}}=d$ and $C_{\mathrm{v}}=1-d$, respectively. By substituting the actual pellet size ( $w=l=8.86 \mathrm{~mm}$ and $h=1.0 \mathrm{~mm}$ ) for $K$ in the Eqs. (1) and (2), the electrical resistivity and the Seebeck coefficient for the pellet are estimated as

$\rho_{\text {cal }}=\rho_{\mathrm{ox}}\left[1-(1-d)^{0.4733}\right]+\frac{\rho_{\mathrm{ox}}}{1-(1-d)^{0.5267}}(1-d)^{0.4733}$

$\alpha_{\mathrm{cal}}=\alpha_{\mathrm{ox}}$

where $\alpha_{\mathrm{A}}, \alpha_{\mathrm{B}}, \rho_{\mathrm{A}}$, and $\rho_{\mathrm{B}}$ in Eqs. (1) and (2) were substituted by $\alpha_{\mathrm{ox}}, \alpha_{\mathrm{v}}=0, \rho_{\mathrm{ox}}$, and $\rho_{\mathrm{v}}=\infty$, respectively. At the second step, the oxide element is simply assumed to be the complex composed of two kinds of elements whose $a-b$ planes are aligned parallel to and perpendicular to the measured direction $y$. The volume fractions of the parallel and the perpendicular elements are approximately assumed to be $C_{/ /}$ 
$=(2+f) / 3$ and $C \perp=(1-f) / 3$, respectively, by using the degree of orientation $f$. By the assumption of the cubic oxide element, the electrical resistivity and the Seebeck coefficient for the oxide elements are estimated as

$\rho_{\mathrm{ox}}=\rho_{\|}\left[1-\left(\frac{1-f}{3}\right)^{1 / 3}\right]+\frac{\rho_{\perp} \rho_{\|}}{\rho_{\perp}\left[1-\left(\frac{1-f}{3}\right)^{2 / 3}\right]+\rho_{\|}\left(\frac{1-f}{3}\right)^{2 / 3}}\left(\frac{1-f}{3}\right)^{1 / 3}$,

$\alpha_{\mathrm{ox}}=\alpha_{\|}\left[1-\left(\frac{1-f}{3}\right)^{1 / 3}\right]+\frac{\alpha_{\|} \rho_{\perp}\left[1-\left(\frac{1-f}{3}\right)^{2 / 3}\right]+\alpha_{\perp} \rho_{\|}\left(\frac{1-f}{3}\right)^{2 / 3}}{\rho_{\perp}\left[1-\left(\frac{1-f}{3}\right)^{2 / 3}\right]+\rho_{\|}\left(\frac{1-f}{3}\right)^{2 / 3}}\left(\frac{1-f}{3}\right)^{1 / 3}$,

where $\alpha_{\mathrm{A}}, \alpha_{\mathrm{B}}, \rho_{\mathrm{A}}$, and $\rho_{\mathrm{B}}$ in Eqs. (1) and (2) were substituted by $\alpha_{/ /}, \alpha \perp, \rho_{/ /}$, and $\rho \perp$, respectively. $\rho_{\text {cal }}$ and $\alpha_{\text {cal }}$ can be calculated when $f$ and $d$ in Fig. 3 (b) are substituted for Eqs. (3)-(6) and $\rho_{\|}=5.7 \mathrm{~m} \Omega \mathrm{cm}, \rho \perp=$ $22.7 \mathrm{~m} \Omega \mathrm{cm}, \alpha_{\|}=113.9 \mu \mathrm{V} / \mathrm{K}$, and $\alpha \perp=53.0 \mu \mathrm{V} / \mathrm{K}$, which were reported for non-doped $\mathrm{Ca}_{3} \mathrm{Co}_{4} \mathrm{O}_{9}$ single crystal at room temperature in Ref. 6, are substituted for them. $\rho_{\text {cal }}$ and $\alpha_{\text {cal }}$ are shown as $\diamond$ and $\diamond$ (and smoothed dashed lines) in Figs. 4(a) and (b). $\rho_{\text {cal }}$ decreases and $\alpha_{\text {cal }}$ slightly increases with the increase in $f$ and $d$ in analogy with $\rho_{\exp }$ and $\alpha_{\exp } . \rho_{\exp }$ is slightly larger than $\rho_{\text {cal }}$ over the present range of $d$ and $f$. This is because the grain boundaries or the crystal defects are not considered in the calculation. $\alpha_{\exp }$ is slightly larger than $\alpha_{\text {cal }}$ over the present range of $d$ and $f$. This is because the effects of Sr addition on the increase in $\alpha$ are not considered in the calculation. Although the absolute values slightly deviate, the calculations can reproduce the relative change of $\rho_{\exp }$ and $\alpha_{\text {exp }}$ with the change of $d$ and $f$.

This suggests that the complex model composed of the void, the parallel, and perpendicular oxide elements well explains the dependence of the thermoelectric properties for the sintered $\mathrm{Ca}_{3} \mathrm{Co}_{4} \mathrm{O}_{9}$ pellet on the compression pressure.

\section{Conclusions}

Sr doped misfit cobalt oxide $\mathrm{Ca}_{2.7} \mathrm{Sr}_{0.3} \mathrm{Co}_{4} \mathrm{O}_{9}$ was prepared by solid-phase reaction, sequent uniaxial compression molding and sintering at $1173 \mathrm{~K}$ for $20 \mathrm{~h}$. The thermoelectric properties were measured with change of the compression pressure of 150-900 MPa in the uniaxial molding. The XRD measurements and the Archimedian experiments revealed that the degree of orientation $f$ and the relative density $d$ of the sintered sample increase with the increase in the compression pressure. $\alpha_{\mathrm{exp}}, Z T$, and $P F$ increase and 
$\rho_{\text {exp }}$ decreases with the increase in the compression pressure owing to the increase in $f$ and $d$. $\alpha_{\text {cal }}$ and $\rho_{\text {cal }}$ as functions of $f$ and $d$ are calculated by the complex model, which composed of the void element, two kinds of oxide elements with different orientations. The calculations can reproduce the relative change of $\rho_{\text {exp }}$ and $\alpha_{\text {exp }}$ with the change of $d$ and $f$.

\section{References}

1. A.C. Masset, C. Michel, A. Maignan, M. Hervieu, O. Toulemonde, F. Studer, B. Raveau, Phys. Rev. B 62, 166 (2000).

2. Y. Miyazaki, K. Kudo, M. Akoshima, Y. Ono, Y. Koike, T. Kajitani, Jpn. J. Appl. Phys. 39, L531 (2000).

3. G. D. Tang, H. H. Guo, T. Yang, D. W. Zang, X. N. Xu, L. Y. Wang, Z. H. Wang, H. H. Wen, Z. D. Zang, and Y. W. Du, Appl. Phys. Lett. 98, 202109 (2011).

4. D. Kenfaui, D. Chateigner, M. Gomina, and J. G. Noudem, Int. J. Appl. Ceram. Technol. 8, 214 (2011).

5. E. Guilmeau, M. Pollet, D. Grebille, D. Chateigner, B. Vertruyen, R. Cloots, R. Funahashi, and B. Ouladiaff, Mater. Res. Bull. 43, 394 (2008).

6. B. C. Zhao, Y. P. Sun, W. J. Lu, X. B. Zhu, and W. H. Song, Phys. Rev. B 74, 144417 (2006).

7. T. Morimura, Y. Sato, Y. Ikuhara, T. Yamaguchi, and M. Hasaka, J. J. Appl. Phys. 52, 071101 (2013).

8. S. Li, R. Funahashi, I. Matsubara, H. Yamada, K. Ueno, and S. Sodeoka, Ceram. Int. 27, 321 (2001).

9. D. Pelloquin, S. Hebert, A. Maignan, and B. Raveau, Solid State Sci. 6, 167 (2004).

10. T. Kajitani, K. Yubuta, X. Huang, and Y. Miyazaki, J. Electron. Mater. 38, 1462 (2009).

11. L.B. Wang, A. Maignan, D. Pelloquin, S. Hebert, and B. Raveau, J. Appl. Phys. 92, 124 (2002).

12. C. Xia, J. Sugiyama, H. Itahara, and T. Tani, J. Cryst. Grow. 276, 519 (2005).

13. I. Matsubara, R. Funahashi, and M. Shikano, Appl. Phys. Lett. 80, 4729 (2002).

14. R. Venkatasubramanian, E. Siivola, T. Colpitts and B. O’Quinn, Nature 413, 597 (2001).

15. F. K. Lotgering J. Inog. Nucl. Chem. 9, 113 (1959). 
16. T. Watanabe, M, Hasaka, and T. Miyase, J. Japan Inst. Metals 58, 353 (1994).

17. T. Watanabe, and M, Hasaka, J. Japan Inst. Metals 63, 508 (1999).

\section{Figure captions}

Fig. 1. Seebeck coefficient $\alpha_{\exp }(\bigcirc)$ and the electrical resistivity $\rho_{\exp }(\mathbf{O})(a)$, and dimensionless figure of merit $Z T(\bigcirc)$ and power factor $P F(\bigcirc)$ (b) measured at room temperature as a function of the compression pressure in the uniaxial molding.

Fig. 2. Dimensionless figure of merit $Z T$ as a function of the measured temperature for various compression pressures in uniaxial molding.

Fig. 3. X-ray diffraction patterns of $\mathrm{Ca}_{2.7} \mathrm{Sr}_{0.3} \mathrm{Co}_{4} \mathrm{O}_{9}$ for the powder sample and the pellet samples molded at 150-900 MPa (a) and degree of orientation $f(\mathbf{O})$ and relative density $d(\bigcirc)$ of sintered pellet as a function of the compression pressure (b).

Fig. 4. Seebeck coefficient $\alpha_{\exp }(\bigcirc)$ and electrical resistivity $\rho_{\exp }(\mathbf{O})$ measured at room temperature as a function of the degree of orientation $f(a)$ and of the relative density $d$ (b) of the pellet samples (solid lines). Seebeck coefficient $\alpha_{\text {cal }}(\diamond)$ and electrical resistivity $\rho_{\text {cal }}(\diamond)$ calculated by complex model as a function of the degree of orientation $f(a)$ and the relative density $d$ (b) of the pellet samples (dashed lines).

Fig. 5. Scheme of complex model composed of A and B elements, which are randomly distributed. Seebeck coefficient $\alpha_{\mathrm{cal}}$ and electrical resistivity $\rho_{\mathrm{cal}}$ are calculated along $y$-axis by the complex model. 

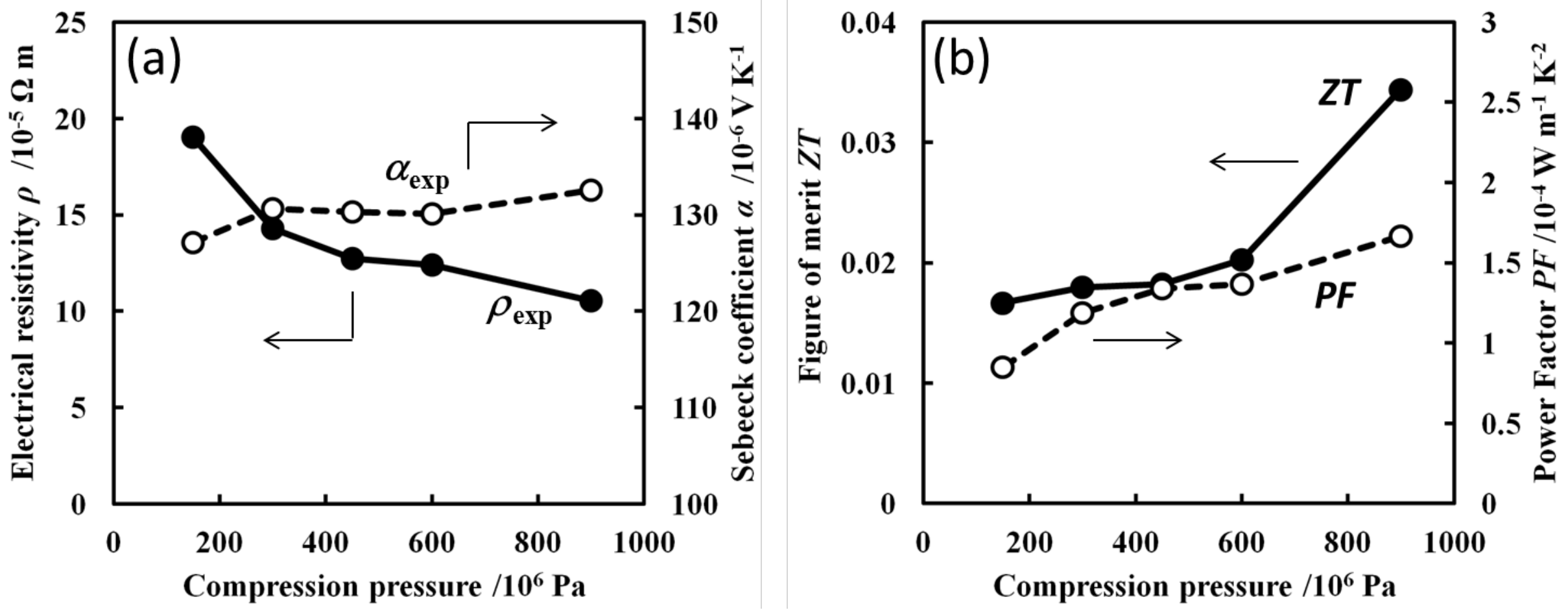

Fig. 1 


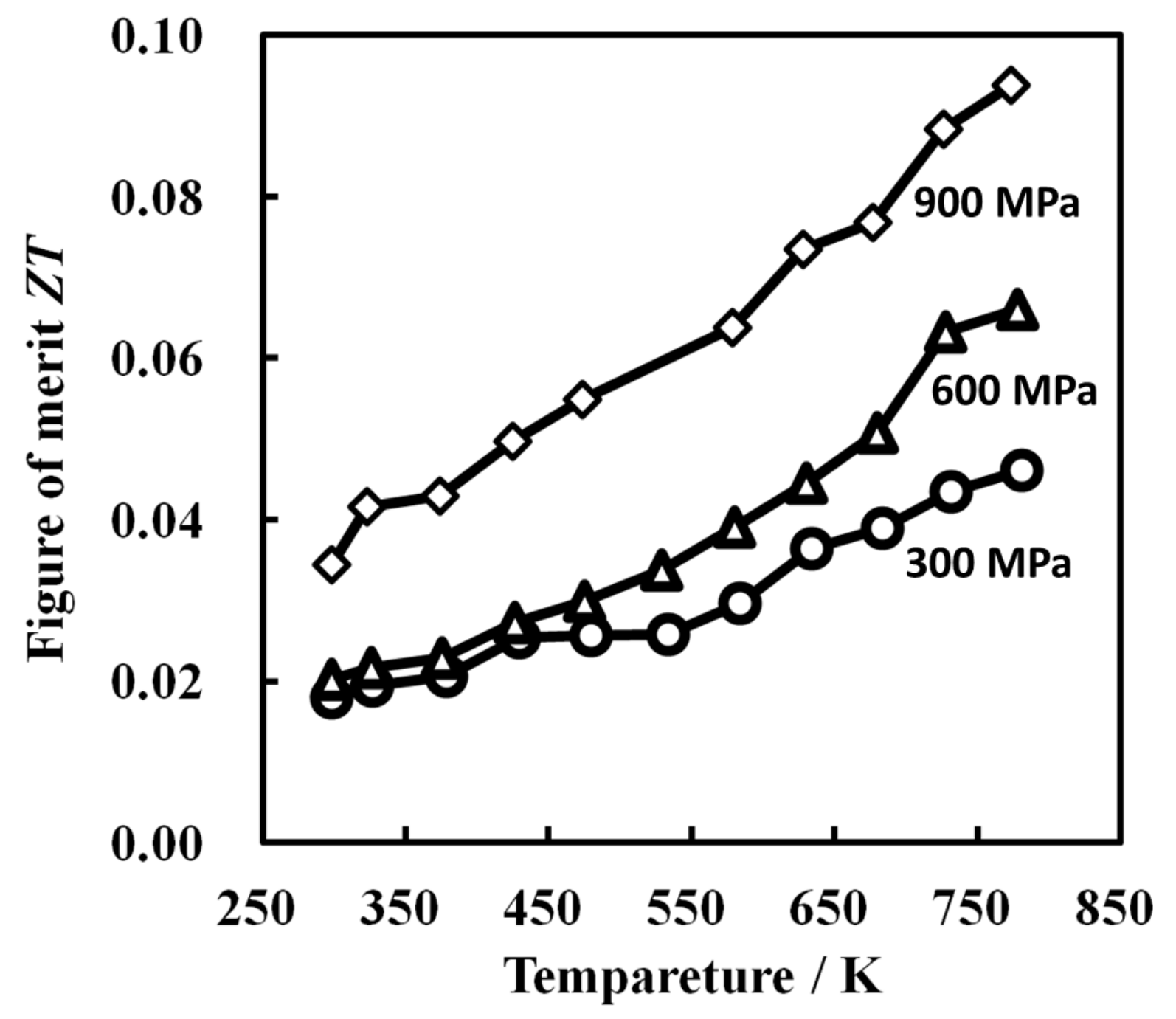

Fig. 2 
(a)

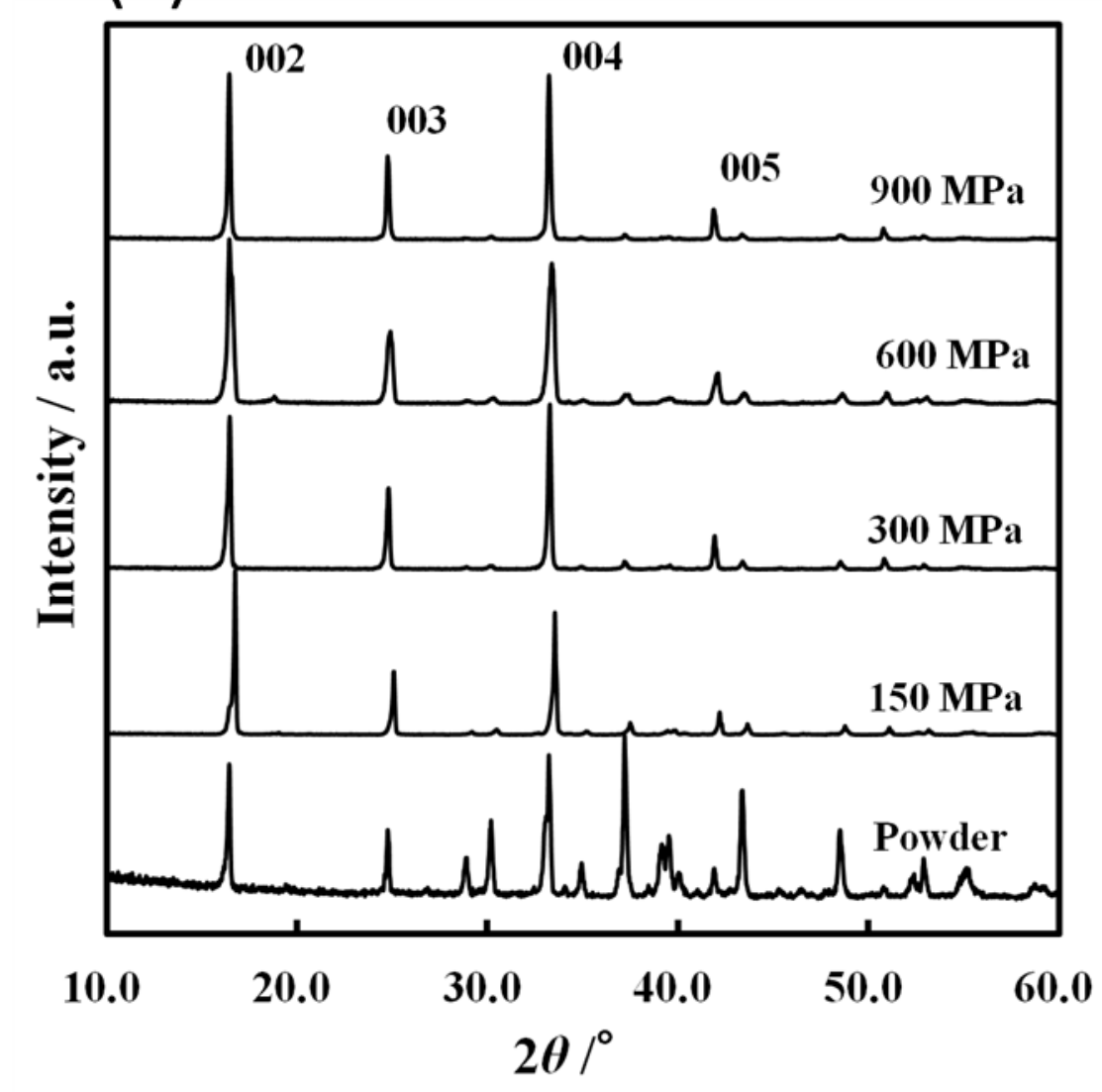

(b)

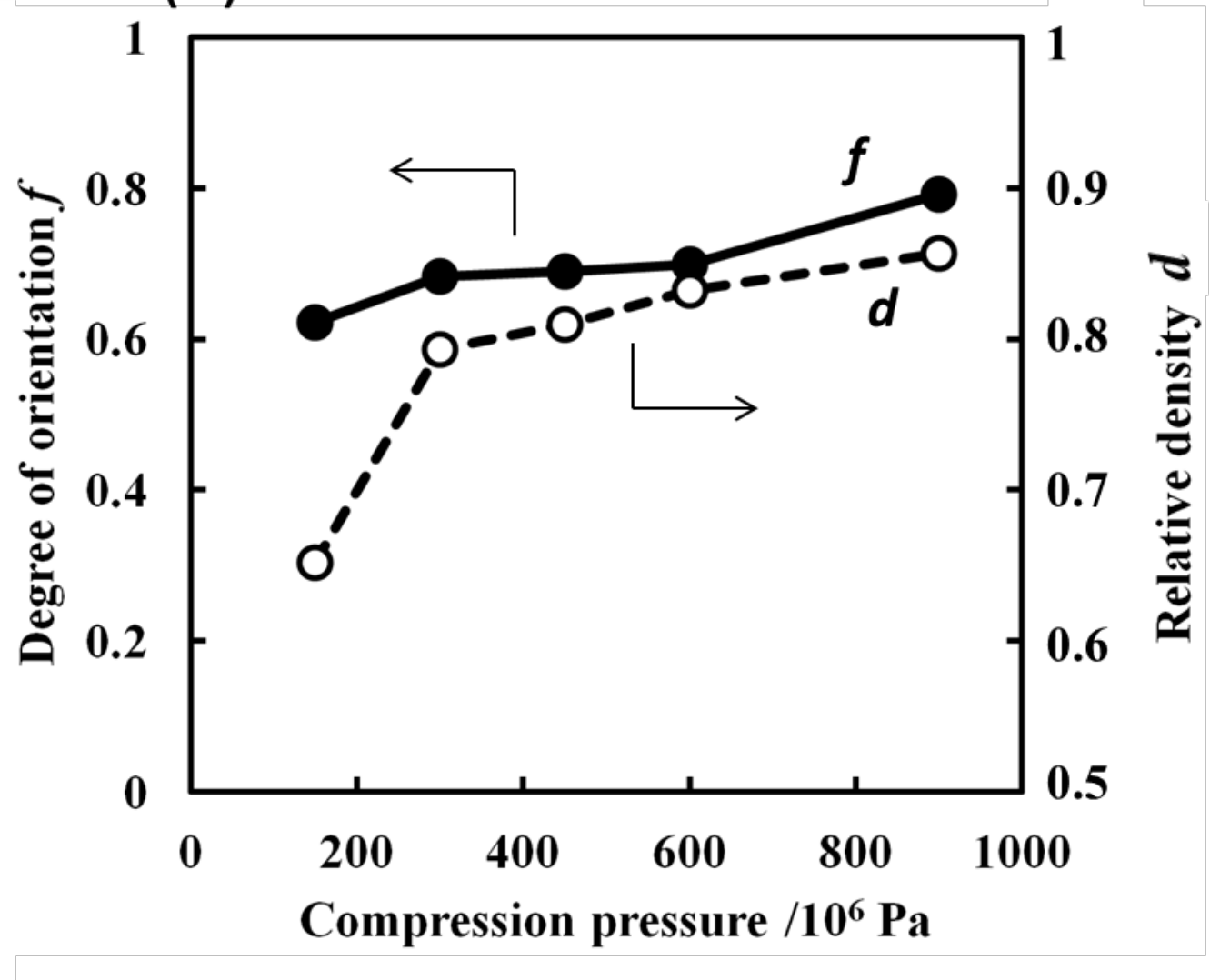

Fig. 3 

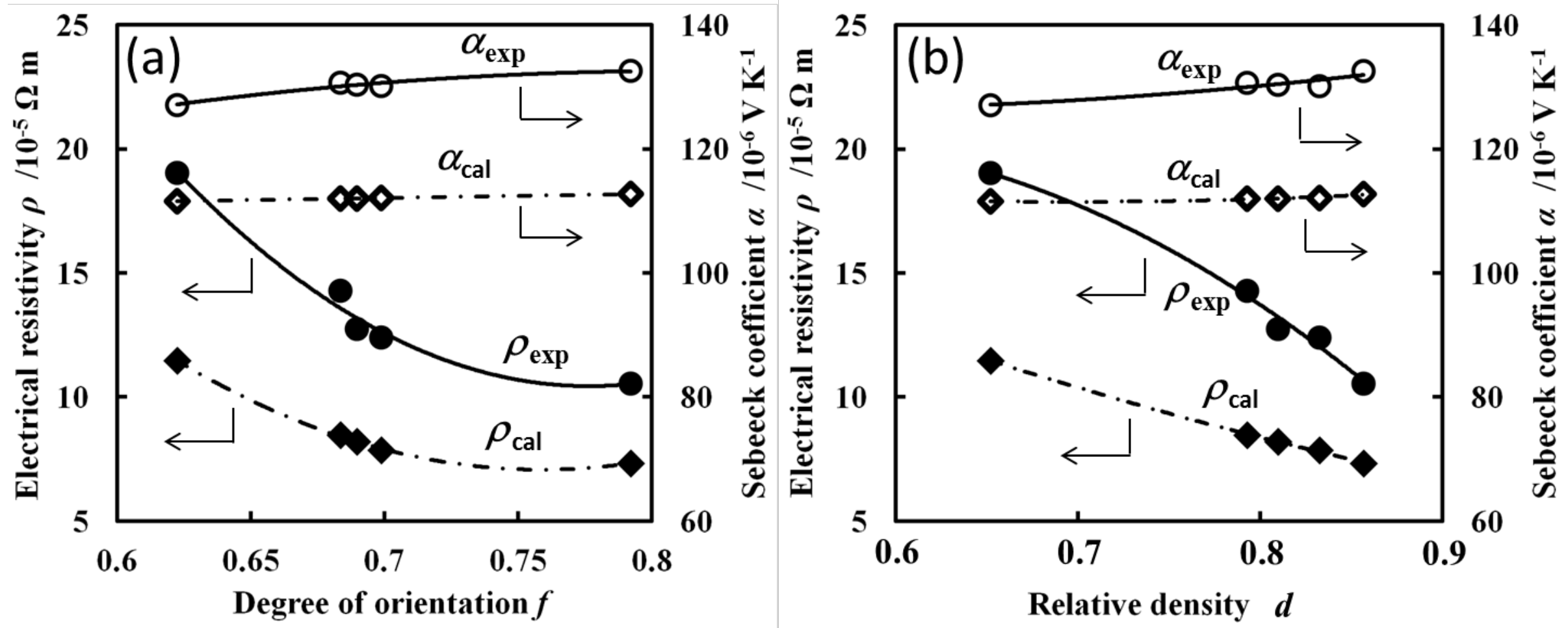

Fig. 4 


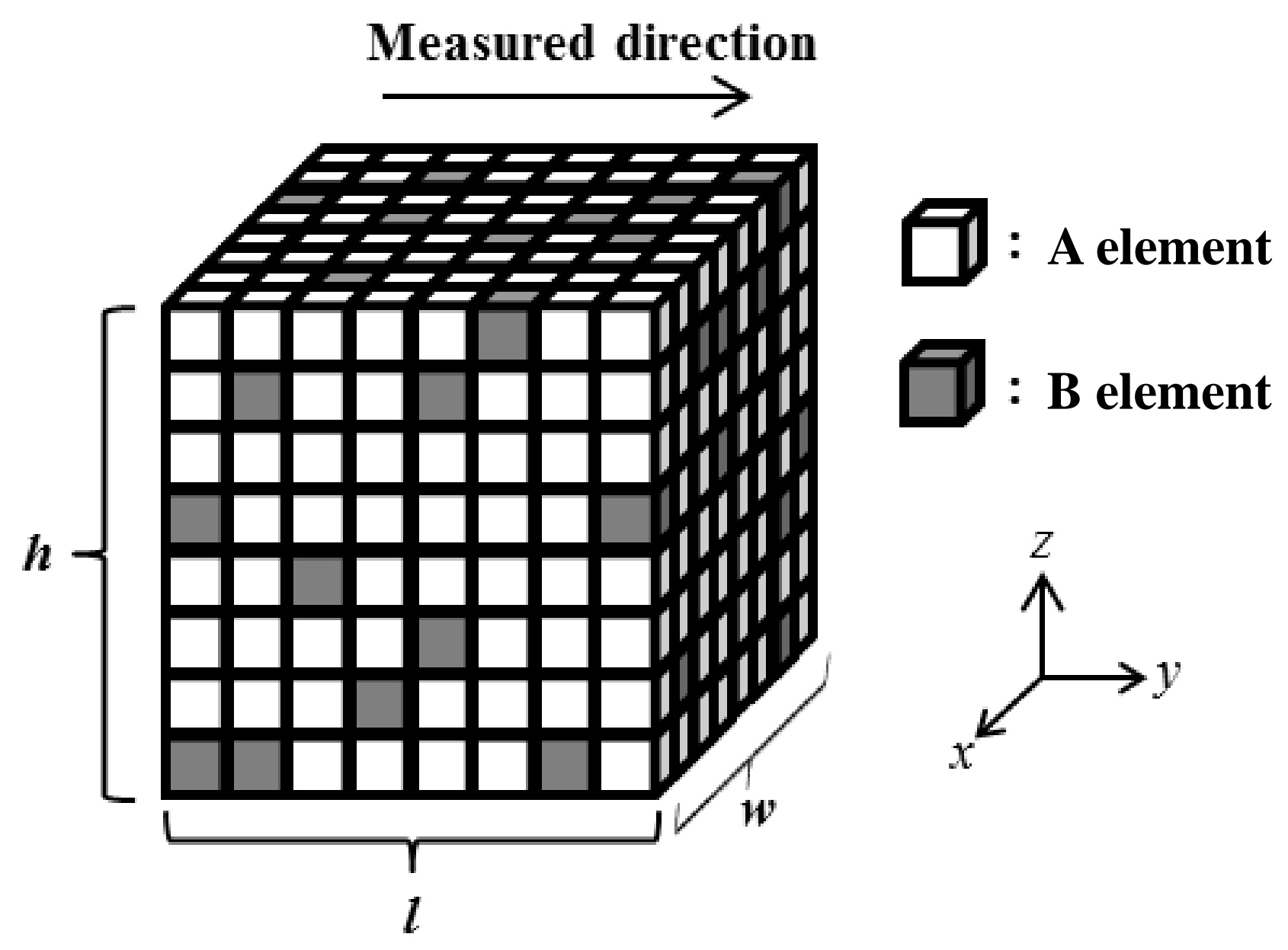

Fig. 5 